\title{
Denying renal transplantation to an adolescent medical cannabis user: An ethical case study
}

\author{
Jennie E. Ryan ${ }^{1,2}$ (D) | Maia Noeder ${ }^{2}$ | Christine Burke ${ }^{2}$ | Samuel C. Stubblefield ${ }^{2,3}$ (D) | \\ Salwa Sulieman $^{2,3}$ | Elissa G. Miller ${ }^{2,3}$ (D)
}

${ }^{1}$ Center for the Study of Drugs, Alcohol, Smoking and Health, School of Nursing, University of Michigan, Ann Arbor, Michigan

${ }^{2}$ Nemours/Al duPont Hospital for Children, Wilmington, Delaware

${ }^{3}$ Sidney Kimmel Medical College, Thomas Jefferson University, Philadelphia,

Pennsylvania

\section{Correspondence}

Jennie E. Ryan, Center for the Study of Drugs, Alcohol, Smoking and Health, School of Nursing, University of Michigan, 400 North Ingalls Building, Office 2234, Ann Arbor, MI 48109-5482.

Email: jennieer@umich.edu

\begin{abstract}
Medical cannabis is now legal in over half of the United States. As more patients adopt this unconventional therapy, it is inevitable that potential transplant recipients will disclose their cannabis use during transplant evaluation. Transplant teams are tasked with the decision to utilize a pressure resource, often with little guidance from international and national professional organizations. Many healthcare providers remain uniformed or misinformed about the risks of cannabis use and organ transplantation. In order to illustrate the multifaceted and complex evaluation of transplant patients using medical cannabis, this article presents the case of a 20 -year-old woman recommended for renal transplant who was originally denied active listing due to her medical cannabis use. A review of the literature explores the perceived and actual risks of cannabis use in the immunocompromised patient. Furthermore, a discussion of the ethics of medical cannabis use and organ transplantation is included with recommendations for multidisciplinary transplant teams.
\end{abstract}

\section{KEYWORDS}

cannabis, ethics, graft risk, medical marijuana, pediatrics, renal transplantation

\section{INTRODUCTION}

Medical cannabis is now legal in 33 states, the District of Columbia, Guam, and Puerto Rico. ${ }^{1}$ An estimated 2.5 million Americans use cannabis for relief of a variety of illnesses. ${ }^{2}$ As more patients adopt this unconventional therapy, healthcare providers will inevitably come across potential transplant recipients utilizing this therapeutic modality. Transplant providers, from multidisciplinary teams, are uniquely impacted by this changing environment. ${ }^{3}$ Providers are tasked with the decision to utilize a precious resource, taking into account an individual's long-term risk. Healthcare providers must take on this matter with little guidance from the healthcare literature or professional organizations. The International Society for Heart and Lung Transplant, a leading source of transplant guidance, recommends that "patients who remain active substance abusers...should not receive heart transplantation." However, when addressing the use of cannabis the recommendation is more ambivalent, stating that "it is at best an issue for which no clear direction exists" and that "each center will need to develop its own specific criteria for adjudicating candidacy for marijuana users". ${ }^{4}$

To explore this issue, we present the case of a 20 -year-old patient followed in our pediatric institution, who was recommended to the transplant team for renal transplantation and had delay of active listing secondary to her medical cannabis use.

Abbreviations: CAMS, childhood anxiety multimodal study; CB2, cannabinoid 2 receptors; CBD, cannabidiol; CBT, cognitive behavioral therapy; CMS, Centers for Medicaid and Medicare Services; DEA, Drug Enforcement Agency; FDA, United States Food and Drug Administration; GVHD, graft-vs-host disease; HR, hazard ratio; MDRD GFR, modification of diet in renal disease glomerular filtration rate; mg, milligrams; OTC, over the counter; PCP, phencyclidine; SSRIs, selective serotonin reuptake inhibitors; THC, tetrahydrocannabinol; VAMS, Visual Analogue Mood Scale; VAS, visual analogue scale. 


\section{2 | DESCRIPTION OF CASE}

The patient is 20 years old with chronic kidney disease stage $\mathrm{V}$ secondary to interstitial fibrosis and glomerulosclerosis. She was recommended for renal transplant by the nephrology team on January 11, 2017. Evaluation for transplantation includes a multidisciplinary assessment by the physician and surgical teams, anesthesia, registered nurses and nurse practitioners, dieticians, social work, and psychology. During her evaluation with the team psychologist, the patient disclosed a long-standing history of depression and anxiety, beginning in early childhood but becoming unmanageable at approximately age 13 (roughly coinciding with the onset of her renal disease). Anxiety generally manifested in worries about the health of family members and occasional anxiety attacks while depressive symptoms (dysphoria, fatigue, avolition, poor concentration, and poor appetite) were triggered by life events such as relationship breakups, family stressors, and changes in her medical status. When symptoms became unmanageable at age 13, the patient began abusing "triple C's" (Coricidin ${ }^{\circledR}$ Cough \& Cold; active ingredients: chlorpheniramine maleate $4 \mathrm{mg}$, dextromethorphan hydrobromide $30 \mathrm{mg}$ ) approximately once a week. She transitioned to weekly alcohol consumption at age 15 followed by regular cannabis use at age 16 . The patient reported that she ceased all alcohol and drug use aside from cannabis at that time, as cannabis managed her anxiety and depression symptoms effectively without the need for additional substances. She had never sought psychiatric care, citing a fear of the side effects of psychotropic medications; she participated in three sessions of psychotherapy at age 17 but terminated treatment due to perceived lack of effectiveness.

At the time of the psychosocial transplant evaluation, the patient reported regular evening/weekend cannabis use and effective management of anxiety and depression symptoms under her current regimen. The patient described her use as medicinal rather than recreational, and evaluation by the transplant psychologist did not yield any concern for impairment in psychosocial functioning while actively using cannabis to manage anxiety and depression symptoms. She was asked to obtain an additional evaluation with an adult psychiatrist to explore psychotropic medication alternatives to cannabis. Psychiatric evaluation determined that the patient's depression and anxiety were "mild" and did not interfere with her functioning, understanding, or decision-making capacity. The potential benefits of SSRIs for symptom relief were discussed as well as adverse side effects of cannabis use but the patient refused a medication trial and continued to express a preference for cannabis management.

The patient had no other physical or social concerns that made her ineligible for renal transplantation. She had routinely demonstrated a high level of maturity and understanding of her chronic condition, and she had been compliant with all of her medical care and had taken ownership of her health care. The patient was deemed an appropriate candidate for renal transplant by the transplant team on September 29, 2017; however, she was listed status seven due to continued concerns regarding her medical cannabis use. The transplant team agreed to list her as inactive until she obtained a legal medical cannabis card in her state, a process which took several months. Once the patient had obtained a medical cannabis card, she could obtain cannabis through a local dispensary, as opposed to peers through the black market. The transplant team felt that cannabis obtained through a dispensary would be safer for consumption.

\section{3 | CONCERNS VOICED BY THE TRANSPLANT TEAM}

Several concerns were voiced by the transplant team regarding the patient's cannabis use and its risk to post-transplant morbidity and mortality. The two primary concerns were risk of Aspergillus infection and risk of cannabis contamination with other illicit substances such as PCP. One healthcare provider also dissuaded the patient from using medical cannabis while encouraging pharmaceutical treatments for management of her anxiety. A thorough review of the literature was performed to determine the scientific merit of these concerns.

\section{1 | Risk of Aspergillus infection}

Risk of infection is an obvious concern in all patients who are immunocompromised and that concern is often heightened with the use of cannabis. Cannabis sativa, whose flowering portion contains multiple cannabinoids, has a symbiotic relationship with multiple microbes, which, like most plants, occurs mostly at the root level. ${ }^{5}$ However, whether the exposure to the natural flora of the cannabis plant causes an increased risk of infection to patients who are immunosuppressed is unknown. The main concern voiced by the transplant team was risk of Aspergillus infection. Since 1988, there have only been two documented case reports of Aspergillus infection in transplant recipients that were associated with cannabis use. ${ }^{6,7}$ When that search was expanded to include all immunocompromised patients, it revealed only one more case report. ${ }^{8}$ There has been one case series of 13 patients with pulmonary Aspergillus infection and human immunodeficiency virus, in which four patients' infections were possibly associated with cannabis use; however, other predisposing factors included neutropenia (due to the disease, to drugs toxic to bone marrow, or both), corticosteroid therapy, previous Pneumocystic jirovecii pneumonia and cytomegalovirus disease, and broad-spectrum antibiotics, including those used in prophylaxis for $P$ jirovecci pneumonia. ${ }^{9}$ Given the growing number of both medicinal and recreational cannabis users in the United States, we can only assume that increasing use would result in increasing reports of Aspergillus infection in immunocompromised patients, but that is not the case. There have been no reports of Aspergillus infection associated with cannabis use since 2001, which may be in part due to advances in sterilization. Ruchlemer et $\mathrm{al}^{10}$ showed that systemic sterilization of medical cannabis can eliminate the risk of opportunistic infections in at-risk patients. 
However, inhalation of cannabis, via smoking and vaporization, can still cause harm to immunosuppressed patients. Substantial evidence has shown a statistical association between cannabis smoking and more frequent chronic bronchitis episodes. ${ }^{11}$ Vaporization is an increasingly popular mode of delivery among medical cannabis patients. ${ }^{12}$ Vaporization does not heat the marijuana to the point of combustion, therefore exposing users to significantly lower levels of toxicants. ${ }^{13}$ Although vaporization reduces the risk of inhaling smoke by-products such as tar, it may expose the patient to lipid inhalation. There is one case report of a renal transplant patient who presented with exogenous lipid pneumonia which was considered to be secondary to inhaling the vapors of a cannabis, isopropyl alcohol, and oil-based mixture that the patient had been using 2-10 times a day for over 10 years. ${ }^{14}$ However, this a rare case of home-prepared cannabis oil used frequently over many years, and it is unclear whether manufactured vaporization cannabis oils and delivery products carry the same risk. Still, edible cannabis administration may be considered a safer route of administration for immunocompromised patients. ${ }^{10}$

The long-term risk of medical cannabis use in renal transplant patients was examined recently by Greenan et al. ${ }^{15}$ In a retrospective review of 1225 renal transplant recipients, the researchers found medical cannabis use was not associated with poorer patient or kidney allograft outcomes at 1 year. The primary outcome of survival at 1-year post-transplant did not differ between users and non-users (100\% vs $97.7 \% ; P=0.622)$. In addition, the proportion of failed allografts, defined as MDRD GFR $<20 \mathrm{~mL} / \mathrm{min}$, did not differ between groups ( $19.7 \%$ vs $17.4 \% ; P=0.62$ ). Ranney et $\mathrm{al}^{16}$ performed another retrospective study done in adult patients with chronic liver disease being evaluated for transplantation over an 8-year time frame (1999 to 2007). The study compared survival between cannabis users and cannabis non-users. On a Kaplan-Meier survival curve, there was not a significant difference in unadjusted patient survival rates in cannabis users vs cannabis non-users. Also, the independent effects of cannabis detection in patients with chronic liver disease were assessed and did not show a significantly higher hazard of mortality (HR $1.09,95 \% \mathrm{Cl}$ $0.78-1.54){ }^{16}$

We found a lack of evidence to support risk of infection from cannabis smoking as a contraindication for renal transplantation. On the contrary, novel research now suggests that cannabis may be used as therapeutic agent for transplant patients. Cannabis has been a target of possible therapeutic use in transplantation due to the known expression of CB2 receptors on immune cells and end organs. Findings from preclinical studies show a role for CB2 receptors in protecting against ischemic/reperfusion injury. ${ }^{17,18}$ Furthermore, a recent phase II clinical trial that examined the combination of CBD with standard therapies for GVHD prophylaxis showed a statistically significant decrease in GVHD rates in CBD users (HR 0.3, $P=0.0002$ ). ${ }^{19}$ The endocannabinoid system shows promise as a possible target for therapies to reduce the immune response, with particularly beneficial outcomes for transplant patients. $^{3}$

\subsection{Cannabis contamination with other illicit substances}

Members of the transplant team voiced a concern that cannabis the patient purchased from sources other than state-run dispensaries could be contaminated with PCP. Marijuana that is soaked in PCP is commonly referred to as "wet," "fry," or "illy." While emergency room visits for PCP intoxication have increased significantly in the United States in the past 10 years, ${ }^{20}$ we could not find a single case study in the healthcare literature that reports an individual presenting to the emergency room with accidental or unintentional PCP intoxication from cannabis contamination. Studies examining the multiple detrimental effects of PCP contaminated cannabis have only identified individuals purposefully ingesting the drug, ${ }^{20}$ and they do not mention any accidental ingestion. An expanded search of popular media also did not find significant case series of individuals presenting with accidental ingestion of PCP contaminated cannabis.

Many healthcare professionals may have apprehension that cannabis procured from sources other than state dispensaries will result in unintentional exposure to more harmful substances. However, this concern is not substantiated with established evidence. There has been only one published case series of illegal cannabis adulterated with lead in Germany in 2008. ${ }^{21}$ Despite common misconceptions, there have been no published case reports of cannabis being contaminated or "laced" with other illicit substances, such as fentanyl. Currently, a large and serious threat to public health is the contamination of illicit heroin, cocaine, methamphetamine, and counterfeit pills with the powerful narcotic fentanyl, with several warnings being issued by the DEA. ${ }^{22}$ However, there have been no substantiated reports of cannabis being laced with fentanyl. Furthermore, a majority of cannabis users who buy product from non-state sources obtain their cannabis from a trusted source such as a friend, therefore bypassing concerns about quality and safety of the product. ${ }^{23}$

\section{4 | TRADITIONAL ANTI-ANXIETY TREATMENTS VS CANNABIS}

During her psychosocial evaluation with the team psychologist, the patient was educated on empirically supported anxiety treatment alternatives to cannabis. CBT is a gold standard, short-term psychotherapy approach that targets thoughts and behaviors that lead to maladaptive emotional responses. CBT emphasizes the use of exposures and coping skills, such as self-talk and relaxation, to unlearn anxious responses to stimuli. Compared to psychological or pharmacological placebo, CBT is consistently found to be an efficacious intervention. ${ }^{24}$ While the patient was initially open to participating in CBT, she ultimately declined psychotherapy services as her symptoms were well managed with her current cannabis regimen. The team psychologist did not express concern with her decision to forgo treatment as current symptoms did not interfere with her functioning or judgment. 
One member of the transplant team, as well as an external adult psychiatrist, recommended that the patient use traditional pharmaceuticals for management of her anxiety, as opposed to cannabis. This recommendation presents two concerns. First, the patient's preference was for cannabis. The patient reported to several healthcare providers that cannabis was more effective at treating her anxiety and she expressed concern about the possible side effects of psychotropic medications. A competent adult patient with decisional capacity should always maintain the right to choose which medical treatment works best for them. Second, traditional pharmaceuticals used for management of anxiety in pediatric patients, while effective, have significant risks that should not be ignored.

The largest randomized control trial to date evaluating treatment modalities for pediatric anxiety is the CAMS. ${ }^{25}$ The study included three different experimental groups: sertraline only, CBT only, combination therapy, and placebo. The study showed that both CBT and sertraline were effective in reducing childhood anxiety, but combination of the two therapies had a superior response rate. A Cochrane Review from 2009 also showed significantly greater treatment response with medication vs placebo. ${ }^{26}$ However, drug-related adverse events were significantly more frequent in medication group vs placebo and accounted for almost twice as many withdraws. Much attention has been brought to the multiple meta-analyses and systemic reviews which have shown increased risk of suicidality, ${ }^{27,28}$ suicidal ideation, and suicide attempts ${ }^{29}$ in children and adolescents treated with antidepressants. These studies prompted the FDA to place a black box warning on the labels of all antidepressants in 2004. Two years following the black box warning, there was a significant reduction in antidepressant use; $20 \%$ for children, $31 \%$ for adolescents, $21.4 \%$ for young adults, and $14.5 \%$ for adults. ${ }^{30}$ This decrease could reflect changing attitudes of prescribers as well as patients and parents. ${ }^{31}$ It is reasonable that patients and parents, aware of the multiple side effects and risks associated with antidepressants, will be reluctant to adopt this modality for management of anxiety. Furthermore, long-term data regarding safety and efficacy of antidepressants for treatment of anxiety are lacking. ${ }^{26}$

Benzodiazepines are often used as second-line pharmacotherapy for management of acute anxiety; however, there is no controlled evidence for their effectiveness in pediatric patients. ${ }^{26,32}$ Furthermore, there are significant concerns for dependence associated with benzodiazepine use. Benzodiazepines have higher rates of both dependence and mortality then does cannabis. Benzodiazepine use for as little as 3 to 6 weeks, even while adhering to therapeutic doses, is associated with the development of physical dependence, with between $15 \%$ and $44 \%$ of chronic benzodiazepine users experiencing protracted moderate to severe withdrawal symptoms upon cessation including emergent anxiety and depressive symptoms. ${ }^{33-35}$ Cannabis dependence is observed at lower rates, with only $9 \%$ of individuals who use cannabis becoming dependent on it. $^{36,37}$ Furthermore, benzodiazepines have a significantly higher risk of fatal overdose then cannabis. Benzodiazepine overdose has been steadily increasing over the past 10 years. ${ }^{38}$ The latest available data from the National Institute of Health showed that 8791 Americans died from benzodiazepine overdose in 2015, while no Americans died of cannabis overdose. ${ }^{38}$ When recommending a drug to a patient, it is essential that prescribers assess the available data regarding safety and efficacy, and in this case, cannabis was both the safer and more effective drug for this patient.

Because of federal restrictions on cannabis, there are few randomized controlled trials examining the efficacy and safety of cannabis for treatment of anxiety. Several small studies have shown both anxiolytic and anxiogenic effects from cannabis, based on the cannabinoid being studied and the dose at which it was administered. Intravenous THC has been shown to increase anxious scores on the VAS. ${ }^{39}$ Inhaled THC at high concentrations (3.6\%) has been shown to increase anxious scores on VAS, while lower concentrations (1.8\%) had no significant effect on VAS anxious scores. $^{40}$

Low-dose oral THC (7.5 mg) has been shown to produce subjective stress-relieving effects, while higher doses $(12.5 \mathrm{mg}$ ) may increase negative mood. ${ }^{41}$

While THC has shown variable effects on anxiety, CBD has consistently been shown to reduce anxiety. A preliminary double-blind, placebo-controlled cross-over study comparing a single dose of CBD (400 mg) to placebo was conducted in 10 treatmentnaive men with generalized social anxiety disorder. Participants rated their anxiety using the VAMS. There was significantly reduced VAMS scores vs placebo on the anxiety factor $(P<0.001) .{ }^{42}$ Another study investigating the use of CBD for treatment of social anxiety disorder also found significantly decreased VAMS scores vs placebo on the anxiety factor $(P<0.012) .{ }^{43}$ CBD has also been shown to decrease VAS factor anxiety scores after public speaking. ${ }^{44}$ However, all these studies were limited by small sample sizes. Further larger randomized control trials are needed to assess safety and efficacy of various cannabinoids for the treatment of anxiety.

While randomized control trials are lacking, much information has been extrapolated from epidemiological data. Much of the debate regarding the relationship of cannabis and anxiety centers around the idea of "self-medication". In various studies, numerous subjects have reported using cannabis to relax, to cope with stress, and to reduce anxiety. ${ }^{45-51}$ Relaxation and relief of tension have been reported as the most common reasons for using cannabis. ${ }^{47,49,51}$ Individuals living in states with medical cannabis laws self-medicate with cannabis at higher rates than those not living in medical cannabis states $(P=0.02) .{ }^{52}$ These findings suggest that access to cannabis may be an important contributor to therapeutic use.

\section{5 | ETHICAL CONSIDERATIONS}

\section{$5.1 \mid$ Four principles}

This case presented several ethical dilemmas. The four principles of medical ethics include the following: respect for autonomy, beneficence, non-maleficence, and social justice. When applying the principles of autonomy and beneficence to this case, one could make 
the argument that this young woman had the right to choose cannabis (autonomy) and that it is in her best interest to move forward with renal transplantation (beneficence). However, the principle of non-maleficence could be applied to argue against medical cannabis use. Non-maleficence requires that healthcare providers first "do no harm," and that our interventions will not have further devastating outcomes for a patient. This patient had end-stage renal disease, and while a renal transplant would provide her the most optimal outcome, she can live for many years without a renal transplant receiving dialysis. But if the team agreed to proceed with transplant and she developed complications secondary to her cannabis use, she could potentially have a life-threatening outcome. Additionally, this patient engaged in alcohol and OTC medication abuse to manage her anxiety and depression before she transitioned to cannabis use. Requiring her to cease engaging in an effective mood management treatment to become transplant eligible could increase the likelihood of a return to high-risk activities. What the team must ask is if her cannabis use places her at any increased risk as compared to noncannabis users? As discussed in this report, the evidence does not support increased risk. The principle of justice furthers this argument by advocating that this patient be evaluated fair and equally as other non-cannabis users. However, stigma surrounding the use of cannabis often introduces implicit bias from healthcare providers.

The decision by the transplant team to allow active listing for renal transplantation only after the patient enrolled in the statesponsored medical cannabis program settled the concern about contaminated product; however, it raises several ethical concerns surrounding social justice. First, this patient was fortunate to live in a state that had an active medical cannabis law. However, consider if she were in a state that did not have legal access to medical cannabis. Requiring that a medical cannabis user register with a state program prior to active transplant listing creates a disparity between patients in states where this is and is not an option. Furthermore, state-level laws that govern medical cannabis use are idiosyncratic and frequently contradictory. Conditions that would qualify a patient for medical cannabis in one state may not qualify them in other, for example, the use of cannabis to treat medication-resistant opioid addiction. In addition, only 8 out of 33 states allow nurse practitioners to certify a patient for medical cannabis, ${ }^{53}$ which may limit access for many Americans in rural and low socioeconomic communities who rely on nurse practitioners as their primary providers. Finally, state programs often have a significant registration fee, and the cost of cannabis through state dispensaries is greater than from non-state sources. ${ }^{54}$ For patients with chronic kidney disease, this cost differential may prohibit registration with the state medical cannabis program. Discriminating against those who are unable to afford state cannabis programs violates the ethical principle of social justice. The inconsistencies and contradictories of state cannabis laws will require federal action to remediate. While federal law maintains cannabis prohibition, the significant social justice concerns will not be resolved.

While federal prohibition is maintained, hospital systems themselves will be faced with multiple ethical dilemmas related to cannabis use in the inpatient setting. Despite legalization of medical and recreational cannabis in several states, many, but not all, hospitals prohibit the use of cannabis products within their institutions due to the theoretical risk to their CMS certification. Our institution is located in the northeast region of the country and does not permit patients or caregivers to bring cannabis into the hospital, despite its legality in the state. However, at least two other pediatric hospitals in bordering states do allow patients and caregivers to bring cannabis products into the hospital. This again presents a violation to the principle of social justice where access to cannabis products is limited not only by the patient's state, but also the policies of the individual institutions within that state. Recommending a patient transfer their care to an outside hospital that allows cannabis products places a burden on the patient and also fragments their care. It is also unlikely that insurance would approve such a transfer of care, therefore placing the patient and family at financial risk.

\section{2 | Stigma}

Illicit drug use is associated with a high degree of stigma from healthcare providers in a variety of settings. ${ }^{55-57}$ Adult medical cannabis users have reported stigmatization related to their use of cannabis as a medication. ${ }^{58-60}$ Stigma may be related to the "blurred boundary" between medicinal use and recreational use. ${ }^{60}$ During team meetings and discussions regarding this patient, some members of the team referred to the patient's cannabis use as recreational, although she never reported recreational use. Incorrectly labeling a patient as a recreational cannabis user can introduce bias and implicit stigmatization. As healthcare providers, we must recognize our own bias regarding cannabis and assure that it does not interfere with our decision-making.

Beyond stigma, healthcare providers may have legal concerns regarding the recommendation for cannabis use and the administration of cannabis to patients. Physicians and nurse practitioners with DEA certification may fear investigation if they allow their patients to utilize a schedule one drug. Nurses working at the bedside may fear disciplinary actions against their licensure if they administer a schedule one drug. Furthermore, all healthcare providers may be uncomfortable with their patients utilizing an herbal therapy such as cannabis that has significant variability and no established dosing guidelines.

\section{3 | Laws against discrimination for medical cannabis patients}

Several states have enacted legislation prohibiting hospitals from determining a patient's suitability for transplant based solely on the basis of medical cannabis use. California, ${ }^{61}$ Washington, ${ }^{62}$ Illinois, ${ }^{63}$ Arizona, ${ }^{64}$ Delaware, ${ }^{65}$ and New Hampshire ${ }^{66}$ have all expanded their medical cannabis bills to include antidiscriminatory sections, designed to protect medical cannabis patients. However, these laws stipulate that the medical use of cannabis must be recommended by a healthcare provider. Patients using medical cannabis outside the guidance of a healthcare professional may not be entitled to the same protections. 
The patient presented in this case study was not protected under the Delaware Medical Marijuana Act until she received her medical cannabis license.

\section{6 | CONCLUSION}

This case study demonstrates that despite traditional perception, convincing evidence of direct harm to a renal transplant patient by cannabis inhalation or ingestion has yet to be established. When examining the risk vs benefit ratio for our patients in situations such as these, it is imperative that we rely upon the established evidence to direct our decision-making. In this case, the patient's listing status was affected for 5 months and the decision to actively list her was only possible because of local laws and the patient's financial means. While a handful of medical cannabis states have antidiscriminatory legislation to protect medical cannabis patients, most states do not. With the growing number of medical cannabis patients, hospitals and transplant teams will be forced to address the issue of cannabis use and transplantation, often without the guidance of national and international transplant societies. In this case study, the use of a multidisciplinary team aided in the decision-making process; however, an ethics consult was warranted given the complexities of the case. We recommend that transplant patients using medical cannabis be evaluated with the same objectivity as non-cannabis using patients. Given the complexities and challenges of state and federal laws pertaining to medical cannabis, we recommend the use of an ethics consult to aid in the team's decision-making process. The role of an ethics consult can be particularly beneficial in states where medical cannabis remains illegal, and patients are using medical cannabis illicitly. These situations present significant legal concerns for transplant teams and patients who choose to disclose their medical cannabis use or have their use discovered through urine drug screening. As stated prior, the ethical and legal concerns regarding cannabis use and transplant will remain unresolved until state and federal laws are congruent. There is a significant need for further research into the therapeutic benefits of cannabis; however, regulatory barriers, including the classification of cannabis as a schedule I drug, impede the advancement of that research. ${ }^{11}$ We therefore agree with the recommendations of the American Academy of Pediatrics ${ }^{67}$ and the American Nurses Association ${ }^{68}$ to reschedule cannabis to schedule II in order to allow further research into its therapeutic value.

\section{CONFLICT OF INTEREST}

No author has a conflict of interest to report.

\section{AUTHORS' CONTRIBUTIONS}

Jennie E. Ryan: Planned the case study; Jennie E. Ryan, Maia Noeder, Christine Burke, Salwa Sulieman, and Elissa G. Miller: Contributed to the first draft of the manuscript; Samuel C. Stubblefield and all authors: Contributed to further drafts. All authors reviewed and approved the final draft.

\section{ORCID}

Jennie E. Ryan (iD https://orcid.org/0000-0002-9726-3602

Samuel C. Stubblefield (iD https://orcid.org/0000-0001-8547-9687

Elissa G. Miller iD https://orcid.org/0000-0002-4302-1149

\section{REFERENCES}

1. National Conference of State Legislatures. State Medical Marijuana Laws. http://www.ncsl.org/research/health/state-medical-marij uana-laws.aspx. Published November 8, 2018. Accessed December 26, 2018.

2. Marijuana Policy Project. Medical Marijuana patient numbers. https ://www.mpp.org/issues/medical-marijuana/state-by-state-medic al-marijuana-laws/medical-marijuana-patient-numbers/. Published February 15, 2018. Accessed December 26, 2018.

3. Rai H, Winder G. Marijuana use and organ transplantation: a review and implications for clinical practice. Curr Psychiatry Rep. 2017;19(11):91.

4. Mehra M, Canter C, Hannan M, et al. The 2016 International Society for Heart Lung Transplantation listing criteria for heart transplantation: a 10-year update. J Heart Lung Transpl. 2016;35(1):1-23.

5. McKernan K, Spangler J, Helbert $\mathrm{Y}$, et al. Metagenomic analysis of medicinal cannabis samples; pathogenic bacteria, toxigenic fungi, and beneficial microbes grow in culture-based yeast and mold tests. F1000 Res. 2016;5:2471.

6. Hamadeh R, Ardehali A, Locksley RM, York MK. Fatal Aspergillosis associated with smoking contaminated marijuana, in a marrow transplant recipient. Chest. 1988;94(2):432-433.

7. Marks WH, Florence L, Lieberman J, et al. Successfully treated invasive pulmonary Aspergillosis associated with smoking marijuana in a renal transplant recipient. Transplantation. 1996;61(12):1771-1774.

8. Szyper-Kravitz M, Lang R, Manor Y, Lahav M. Early invasive pulmonary Aspergillosis in a leukemia patient linked to Aspergillus contaminated marijuana smoking. Leuk Lymphoma. 2001;42(6):1433-1437.

9. Denning D, Follasbee S, Scolaro M, Norris S, Edelstein H, Stevens D. Pulmonary Aspergillosis in the acquired immunodeficiency syndrome. N Engl J Med. 1991;324:654-662.

10. Ruchlemer R, Amit-Kohn M, Raveh D, Hanuš L. Inhaled medicinal cannabis and the immunocompromised patient. Support Care Cancer. 2015;23(3):819-822.

11. The National Academies of Sciences, Engineering, Medicine. The health effects of cannabis and cannabinoids: the current state of evidence and recommendations for research. http://www.natio nalacademies.org/hmd/Reports/2017/health-effects-of-cannabisand-cannabinoids.aspx. Published January 2017. Accessed March 8, 2017.

12. Shiplo S, Asbridge M, Leatherdale ST, Hammond D. Medical cannabis use in Canada: vapourization and modes of delivery. Harm Reduct J. 2016;13(1):30. https://doi.org/10.1186/s12954-016-0119-9.

13. Earleywine M, Barnwell SS. Decreased respiratory symptoms in cannabis users who vaporize. Harm Reduct J. 2007;4:11. https://doi. org/10.1186/1477-7517-4-11.

14. Vethanayagam D, Pugsley S, Dunn E, Russell D, Kay J, Allen C. Exogenous lipid pneumonia related to smoking weed oil following cadaveric renal transplantation. Can Respir J. 2000;7(4):338-342.

15. Greenan G, Ahmad SB, Anders MG, Leeser A, Bromberg JS, Niederhaus SV. Recreational marijuana use is not associated 
with worse outcomes after renal transplantation. Clin Transplant. 2016;30(10):1340-1346. https://doi.org/10.1111/ctr.12828.

16. Ranney DN, Acker WB, Al-Holou SN, et al. Marijuana use in potential liver transplant candidates. Am J Transplant. 2009;9(2):280-285. https://doi.org/10.1111/j.1600-6143.2008.02468.x.

17. Hochhauser E, Lahat E, Sultan M, et al. Ultra low dose delta 9-tetrahydrocannabinol protects mouse liver from ischemia reperfusion injury. Cell Physiol Biochem. 2015;36(5):1971-1981.

18. Rijesh M, Pan H, Mukhopadhyay $\mathrm{P}$, et al. Pivotal advance: Cannabinoid-2 receptor agonist HU-308 protects against hepatic ischemia/reperfusion injury by attenuating oxidative stress, inflammatory response, and apoptosis. J Leukoc Biol. 2007;82(6):1382-1389.

19. Yeshurun M, Shpilberg O, Herscovici C, et al. Cannabidiol for the prevention of graft-versus-host-disease after allogeneic hematopoietic cell transplantation: results of a phase II study. Biol Blood Marrow Transplant. 2015;21(10):1770-1775.

20. Emergency Bush DM. Department Visits Involving Phencyclidine (PCP). Substance Abuse and Mental Health Services Administration (US); 2013. https://proxy.library.upenn.edu:2065/books/NBK38 5063/. Accessed June 16, 2018.

21. Busse F, Omidi L, Timper K, et al. Lead poisoning due to adulterated marijuana. N Engl J Med. 2008;358(15):1641-1642.

22. Drug Enforcement Agency. Cocaine laced with fentanyl leads to multiple deaths, overdoses. https://www.dea.gov/press-relea ses/2018/09/14/cocaine-laced-fentanyl-leads-multiple-deathsoverdoses. Accessed December 19, 2018.

23. Parker H, Aldridge J, Measham F. Illegal Leisure: The Normalization of Adolescent Recreational Drug Use. London, UK: Routledge; 1998.

24. Hoffman AJ. Enhancing self-efficacy for optimized patient outcomes through the theory of symptom self-management. Cancer Nurs. 2013;36(1):E16-E26.

25. Walkup JT, Albano AM, Piacentini J, et al. Cognitive behavioral therapy, sertraline, or a combination in childhood anxiety. N Engl J Med. 2008;359:2754-2766.

26. Ipser JC, Stein DJ, Hawkridge S, Hoppe L. Pharmacotherapy for anxiety disorders in children and adolescents. Cochrane Database Syst Rev. 2009;(3):CD005170. https://doi.org/10.1002/14651858

27. Hammad TA, Laughren T, Racoosin J. Suicidality in pediatric patients treated with antidepressant drugs. Arch Gen Psychiatry. 2006;63(3):332-339.

28. Sharma T, Guski LS, Freund N, Gøtzsche PC. Suicidality and aggression during antidepressant treatment: systematic review and metaanalyses based on clinical study reports. Br Med J. 2016;352:i65.

29. Bridge JA, lyengar S, Salary CB, et al. Clinical response and risk for reported suicidal ideation and suicide attempts in pediatric antidepressant treatment: a meta-analysis of randomized controlled trials. JAMA. 2007;297(15):1683-1696.

30. Lu CY, Zhang F, Lakoma MD, et al. Changes in antidepressant use by young people and suicidal behavior after FDA warnings and media coverage: quasi-experimental study. BMJ. 2014;348:g3596.

31. Friedman R. Antidepressants' black-box warning-10 years Later. $N$ Engl J Med. 2014;371:1666-1668.

32. Bushnell G, Compton SN, Dusetzina S, et al. Treating pediatric anxiety: Initial use of SSRIs and other antianxiety prescription medications. J Clin Psychiatry. 2017;79(1):1-19. https://doi.org/10.4088/ JCP.16m11415.

33. Peturson $\mathrm{H}$, Lader M. Withdrawal from long-term benzodiazepine treatment. Br Med J Clin Res Ed. 1981;283(6292):643-645.

34. Ashton H. Protracted withdrawal syndromes from benzodiazepines. J Subst Abuse Treat. 1991;8(1-2):19-28.

35. Kann C, Breteler M, Zitman F. High prevalence of benzodiazepine dependence in out-patient users, based on the DSM-III-R and ICD-10 criteria. Acta Psychiatr Scand. 1997;96(2):85-93.
36. Anthony JC, Warner LA, Kessler RC. Comparative epidemiology of dependence on tobacco, alcohol, controlled substances, and inhalants: Basic findings from the National Comorbidity Survey. Exp Clin Psychopharmacol. 1994;2(3):244-268.

37. Lopez-QuinteroC, de los Cobos et al. Probability and predictors of transition from first use to dependence on nicotine, alcohol, cannabis, and cocaine: results of the National Epidemiologic Survey on Alcohol and Related Conditions (NESARC). Drug Alcohol Depend. 2011;115(1-2):120-130.

38. National Institute on Drug Abuse. National overdose deaths from select prescription and illicit drugs. September 2017. https://www. drugabuse.gov/related-topics/trends-statistics/overdose-deathrates. Accessed December 19, 2018.

39. D'Souza DC, Perry E, MacDougall L, et al. The psychotomimetic effects of intravenous delta-9-tetrahydrocannabinol in healthy individuals: Implications for psychosis. Neuropsychopharmacology. 2004;29(8):1558-1572.

40. Ilan $A B$, Gevins $A$, Coleman $M$, ElSohly $M A$, de Wit $H$. Neurophysiological and subjective profile of marijuana with varying concentrations of cannabinoids. Behav Pharmacol. 2005;16(5-6):487-496.

41. Childs E, Lutz JA, de Wit H. Dose-related effects of delta-9-THC on emotional responses to acute psychosocial stress. Drug Alcohol Depend. 2017;177:136-144.

42. Crippa J, Derenusson GN, Ferrari TB, et al. Neural basis of anxiolytic effects of cannabidiol (CBD) in generalized social anxiety disorder: a preliminary report. J Psychopharmacol (Oxf). 2011;25(1):121-130.

43. Bergamaschi MM, Queiroz R, Chagas $M$, et al. Cannabidiol reduces the anxiety induced by simulated public speaking in treatment-naïve social phobia patients. Neuropsychopharmacology. 2011;36(6):1219-1226.

44. Zuardi AW, Cosme RA, Graeff FG, Guimarães FS. Effects of ipsapirone and cannabidiol on human experimental anxiety. J Psychopharmacol (Oxf). 1993;7(1_suppl):82-88.

45. Crippa JA, Zuardi AW, Martín-Santos R, et al. Cannabis and anxiety: a critical review of the evidence. Hum Psychopharmacol. 2009;24(7):515-523.

46. Bonn-Miller MO, Zvolensky MJ, Bernstein A. Marijuana use motives: Concurrent relations to frequency of past 30-day use and anxiety sensitivity among young adult marijuana smokers. Addict Behav. 2007;32(1):49-62.

47. Boys A, Marsden J, Griffiths P, Fountain J, Stillwell G, Strang J. Substance use among young people: the relationship between perceived functions and intentions. Addiction. 1999;94(7):1043-1050.

48. Buckner JD, Schmidt NB, Lang AR, Small JW, Schlauch RC, Lewinsohn PM. Specificity of social anxiety disorder as a risk factor for alcohol and cannabis dependence. J Psychiatr Res. 2008;42(3):230-239.

49. Hathaway AD. Cannabis effects and dependency concerns in longterm frequent users: a missing piece of the public health puzzle. Addict Res Theory. 2003;11(6):441-458.

50. Ogborne AC, Smart RG, Weber T, Birchmore-Timney C. Who is using cannabis as a medicine and why: an exploratory study. $J$ Psychoactive Drugs. 2000;32(4):435-443.

51. Reilly D, Didcott P, Swift W, Hall W. Long-term cannabis use: characteristics of users in an Australian rural area. Addict Abingdon Engl. 1998;93(6):837-846.

52. Sarvet AL, Wall MM, Keyes KM, Olfson M, Cerdá M, Hasin DS. Self-medication of mood and anxiety disorders with marijuana: Higher in states with medical marijuana laws. Drug Alcohol Depend. 2018;186:10-15

53. Gowen K, Cahill M, Russell K, Cronquist R. Medical Marijuana and nursing practice: current legislation, scientific literature review, and nursing implications. Presented at the National Council 
of State Boards of Nursing. 2018; https://www.ncsbn.org/2018D CM_Medical-Marijuana.pdf.

54. Legal and illegal cannabis price difference by U.S. state 2016. Statista. https://www.statista.com/statistics/589821/street-anddispensary-marijuana-price-difference-by-us-state/. Published January 2016. Accessed August 14, 2018.

55. Henderson S, Stacey CL, Dohan D. Social stigma and the dilemmas of providing care to substance users in a safety-net emergency department. J Health Care Poor Underserved. 2008;19(4):1336-1349.

56. Ahern KJ. Ten tips for reflexive bracketing. Qual Health Res. 1999;9(3):407-411.

57. Room R. Stigma, social inequality and alcohol and drug use. Drug Alcohol Rev. 2005;24:143-155.

58. Bottorff JL, Bissell L, Balneaves LG, Oliffe JL, Capler NR, Buxton J. Perceptions of cannabis as a stigmatized medicine: a qualitative descriptive study. Harm Reduct J. 2013;10:2.

59. Satterlund TD, Lee JP, Moore RS. Stigma among California's medical marijuana patients. J Psychoactive Drugs. 2015;47(1):10-17.

60. Ryan J, Sharts-Hopko N. The experiences of medical marijuana patients: a scoping review of the qualitative literature. J Neurosci Nurs. 2017;49(3):185-190.

61. Assembly Bill No. 258 Organ Transplants: Medical Marijuana: Qualified Patients; 2015. https://leginfo.legislature.ca.gov/faces/ billNavClient.xhtml?bill_xml:id=201520160AB258. Accessed August 14, 2018.

62. Medical Cannabis: Suitability for Organ Transplant; 2017. http:// apps.leg.wa.gov/rcw/default.aspx?cite=69.51a\&full=true. Accessed August 14, 2018.
63. Compassionate Use of Medical Cannabis Pilot Program Act; 2014. http://www.ilga.gov/legislation/ilcs/ilcs3.asp?ActID=3503\&Chapt erID=35. Accessed August 14, 2018.

64. Discrimination Prohibited: Arizona Medical Marijuana Act; 2010. https://www.azleg.gov/ars/36/02813.htm. Accessed August 14, 2018.

65. The Delaware Medical Marijuana Act; 2018. http://www.delco de.delaware.gov/title16/c049a/index.shtml. Accessed August 14, 2018.

66. Therapeutic Cannabis program protections and prohibitions for qualifying patients; 2015. https:/www.dhhs.nh.gov/oos/tcp/ documents/protectionspatient.pdf. Accessed August 14, 2018.

67. American Academy of Pediatrics. The impact of marijuana policies on youth: Clinical, research, and legal update. Pediatrics. 2015;135(3):584-587.

68. American Nurses Association. Therapeutic Use of Marijuana and Related Cannabinoids. Position Statement. Silver Spring, MD: American Nurses Association; 2016.

How to cite this article: Ryan JE, Noeder M, Burke C, Stubblefield SC, Sulieman S, Miller EG. Denying renal transplantation to an adolescent medical cannabis user: An ethical case study. Pediatr Transplant. 2019;23:e13467. https:// doi.org/10.1111/petr.13467 